\title{
FINITE FOUR-GENERATED SIMPLE LATTICES CONTAIN ALL FINITE LATTICES
}

\author{
WERNER POGUNTKE AND IVAN RIVAL
}

\begin{abstract}
ABSTRaCr. Every finite lattice is embeddable in a finite, four-generated, simple lattice.
\end{abstract}

The purpose of this note is to prove

THEOREM. Every finite lattice is embeddable in a finite, four-generated, simple lattice.

In the middle 1940's P. Whitman [7] conjectured that every finite lattice is embeddable in a finite partition lattice. Although this conjecture remains unanswered, it is known that some larger classes of lattices do provide such embeddings. For example, the class of finite geometric lattices contains all finite partition lattices and R. P. Dilworth [1] has shown that every finite lattice is embeddable in a finite geometric lattice. The partition lattice is simple and can be considered as the lattice of subspaces of a suitable geometry. By loosening the conditions on this geometry J. Hartmanis [3], [4] has shown that every finite lattice is embeddable in the finite simple lattice of subspaces of a geometry.

Our result has a similar character. Its motivation is two-fold: first, H. Strietz [6] has proven that every finite partition lattice is four-generated; second, $\mathrm{R}$. Wille [8] has proven that any nontrivial, simple, three-generated lattice is either a two-element chain or the five-element lattice of length two. In particular, the number of generators in the statement of our theorem cannot be improved.

For elements $x, y$ in a partially ordered set, $x$ covers $y$, denoted $x>y$, if $x>z \geqslant y$ implies $z=y$.

LEMMA 1. Let $L$ be a lattice, let $b, c \in L$ with $b>c$, and let $a$ be an element not in $L$. Then $L^{\prime}=L \cup\{a\}$, with the partial ordering induced by $L$ and the comparabilities $b>a>c$, is a lattice. Moreover, $L$ is a sublattice of $L^{\prime}$.

Proof. The proof is routine once we observe that the comparabilities of $a$ in $L^{\prime}$ are determined by the comparabilities of $b$ and $c$ in $L$.

Our proof makes essential use of the following important result due to $R$. A. Dean [2] and, independently, Ju. I. Sorkin [5].

LEMMA 2. Every finite lattice is embeddable in a finite, three-generated lattice.

Received by the editors May 28, 1975.

AMS (MOS) subject classifications (1970). Primary 06A30. 
Let $L$ be a finite lattice and let $T(L)$ be the set of all maximal three-element chains in $L$; that is,

$$
T(L)=\left\{\left(x_{i}, y_{i}, z_{i}\right) \in L^{3} \mid z_{i}>y_{i}>x_{i} \text { for each } i=1,2, \ldots, n\right\} .
$$

Let $\left\{a_{1}, b_{1}\right\},\left\{a_{2}, b_{2}\right\}, \ldots,\left\{a_{n}, b_{n}\right\}$ be a sequence of disjoint pairs of elements, each disjoint also from $L$, and let $L^{\prime}=L \cup \cup_{i=1}^{n}\left\{a_{i}, b_{i}\right\}$ have the partial ordering induced by $L$ and the comparabilities $z_{i}>a_{i}>x_{i}, z_{i}>b_{i}>x_{i}$, for each $i=1,2, \ldots, n$. In view of Lemma $1, L^{\prime}$ is a lattice that contains $L$ as a sublattice. Indeed, since any two minimal nontrivial quotients are projective, $L^{\prime}$ is simple; a fortiori, $L^{\prime}$ is subdirectly irreducible. By Lemma $2, L^{\prime}$ is embeddable in a finite three-generated lattice $L^{\prime \prime}$. As $L^{\prime \prime}$ is a subdirect product of finite, three-generated, subdirectly irreducible lattices, and as $L^{\prime}$ is itself subdirectly irreducible, we conclude that $L$ is embeddable in a finite, threegenerated, subdirectly irreducible lattice $L^{\prime \prime \prime}$. The last step, of embedding $L^{\prime \prime \prime}$ in a finite, four-generated, simple lattice, is accomplished by our final lemma. Let us note that there is no loss in generality to assume that the length of $L^{\prime \prime \prime}$ is at least three.

For elements $a, b$ in a lattice $L$ let $\theta(a, b)$ denote the least congruence relation in $L$ that identifies $a$ and $b$; for quotients $a / b$ and $c / d$ in $L$ we write $a / b \nearrow c / d$ (or $c / d \searrow a / b$ ) whenever $a \vee d=c$ and $a \wedge d=b$. Let 0 and 1 denote the universal bounds of $L$. For all further terminology we refer to [1].

LEMMA 3. Let $L$ be a finite subdirectly irreducible lattice of length at least three. Then there exist $a, b \in L$ with $1>a>b>0$ such that $\theta(a, b)$ is the minimum nontrivial congruence relation in L. Furthermore, for distinct elements $c, d$, disjoint from $L, L^{\prime}=L \cup\{c, d\}$, with the partial ordering induced by $L$ and the comparabilities $1>c>b, c>d>0$, is a simple lattice that contains $L$ as a sublattice.

In $L^{\prime}, c=b \vee d$; hence, if $L$ is three-generated then $L^{\prime}$ is four-generated.

Proof. Let $\theta$ be the minimum nontrivial congruence relation in $L$. If $1>c_{1}$ and $\theta=\theta\left(1, c_{1}\right)$, say, then, as $L$ is subdirectly irreducible, there is a coatom $c_{2}$ in $L$ distinct from $c_{1}$. Furthermore, if there exists $d$ satisfying $c_{1} \succ d$ $>c_{1} \wedge c_{2}\left(\right.$ or $\left.c_{2}>d>c_{1} \wedge c_{2}\right)$ then $\theta=\theta\left(c_{1}, d\right) \quad\left(\theta=\theta\left(c_{2}, d\right)\right)$. Let us suppose that $c_{1}>c_{1} \wedge c_{2}$ and $c_{2}>c_{1} \wedge c_{2}$. If $c_{1} \wedge c_{2}>0$ then $\theta=$ $\theta\left(c_{1}, c_{1} \wedge c_{2}\right)$; if $c_{1} \wedge c_{2}=0$ then, since $L$ has length at least three, there exists a coatom $c_{3}$ distinct from $c_{1}$ and $c_{2}$. In this case, $\left\{0, c_{1}, c_{2}, c_{3}, 1\right\}$ is a sublattice of $L$ and $0 \equiv 1(\theta)$; that is, $L$ is simple. Hence, any $a, b \in L$ satisfying $1>a>b>0$ also satisfy $\theta=\theta(a, b)$.

Now, in the light of Lemma 1, the partially ordered set $L^{\prime}$ as constructed above is a lattice and $L$ is a sublattice of $L^{\prime}$. Moreover, the congruence relation $\theta^{\prime}$ generated by identifying $a$ with $b$ in $L^{\prime}$ is the minimum nontrivial congruence relation in $L^{\prime}$; hence, $L^{\prime}$ is subdirectly irreducible. It remains only to show that $0 \equiv 1 \quad\left(\theta^{\prime}\right)$.

Since $L$ is subdirectly irreducible, $L$ contains distinct coatoms $x_{1}, x_{2}$ and distinct atoms $y_{1}, y_{2}$. If $x_{1}>b$ and $x_{2}>b$ then

$$
a / b \nearrow 1 / c \searrow x_{1} / b \text { and } a / b \nearrow 1 / c \searrow x_{2} / b \text {. }
$$

Hence, $x_{1} \equiv b \quad\left(\theta^{\prime}\right)$ and $x_{2} \equiv b \quad\left(\theta^{\prime}\right)$ so that $1 \equiv b \quad\left(\theta^{\prime}\right)$. If $x_{1} \geqslant b$ then 


$$
a / b \nearrow a / c \searrow x_{1} / x_{1} \wedge b \nearrow 1 / b \text {. }
$$

Therefore, under any circumstances $1 \equiv b \quad\left(\theta^{\prime}\right)$. On the other hand, if $y_{1} \leqslant b$ and $y_{2} \leqslant b$ then

$$
c / b \searrow d / 0 \nearrow c / y_{1} \text { and } c / b \searrow d / 0 \nearrow c / y_{2} \text {. }
$$

Then $y_{1} \equiv c \quad\left(\theta^{\prime}\right)$ and $y_{2} \equiv c \quad\left(\theta^{\prime}\right)$ so that $0 \equiv c \quad\left(\theta^{\prime}\right)$ which, together with $1 \equiv b\left(\theta^{\prime}\right)$ implies that $0 \equiv 1\left(\theta^{\prime}\right)$. Finally, if $y_{1} \$ b$ then $a / b \nearrow 1 / c \searrow y_{1} / 0$ and, as $c / b \searrow d / 0$, we conclude that $y_{1} \equiv 0 \quad\left(\theta^{\prime}\right)$ and $d \equiv 0 \quad\left(\theta^{\prime}\right)$ so that $y_{1} \vee d=1 \equiv 0 \quad\left(\theta^{\prime}\right)$.

\section{REFERENCES}

1. P. Crawley and R. P. Dilworth, Algebraic theory of lattices, Prentice-Hall, Englewood Cliffs, N.J., 1973.

2. R. A. Dean, Component subsets of the free lattice on $n$ generators, Proc. Amer. Math. Soc. 7 (1956), 220-226. MR 18, 6.

3. J. Hartmanis, Two embedding theorems for finite lattices, Proc. Amer. Math. Soc. 7 (1956), 571-577. MR 18, 6.

4. - Lattice theory of generalized partitions, Canad. J. Math. 11 (1959), 97-106. MR 20 \#6367.

5. Ju. I. Sorkin, On the embedding of latticoids in lattices, Dokl. Akad. Nauk. SSSR 95 (1954), 931-934. (Russian) MR 15, 926.

6. H. Strietz, Every finite partition lattice is four-generated, Notices Amer. Math. Soc. 22 (1975), A-507.

7. P. M. Whitman, Lattices, equivalence relations, and subgroups, Bull. Amer. Math. Soc. 52 (1946), 507-522. MR 8, 62.

8. R. Wille, A note on simple lattices, 1975.

Fachbereich Mathematik, Technische Hochschule Darmstadt, Darmstadt Federal REPUBLIC OF GERMANY

Department of Mathematics, University of Calgary, Calgary, Alberta T2N 1N4, CANADA 